\title{
Sentimentos dos estudantes utilizando ensino remoto durante pandemia COVID-19: interferência no processo de aprendizagem
}

\section{Students' feelings using remote learning during the COVID-19 pandemic: interference in the learning process}

Celia Maria Ribeiro de Vasconcelos ${ }^{1}$, Ana Maria Gomes de Oliveira ${ }^{2}$, Carolinne Luiza Cavalcanti da Hora ${ }^{3}$, Gabriellen da Silva Santos ${ }^{4}$, José Igor Pereira Batista ${ }^{5}$, Maria

Fernanda Tavares Lins Oliveira ${ }^{6}$, Ana Karine Laranjeiras de Sá ${ }^{7}$

1. ORCID: http://orcid.org/0000-0001-9309-4795. Doutora. Docente Titular do Instituto Federal de Educação, Ciência e Tecnologia de Pernambuco IFPE - Campus Pesqueira.PE, Brasil. E-mail: celia@pesqueira.ifpe.edu.br

2. ORCID: https://orcid.org/0000-0003-1686-0980. Graduanda em Bacharelado em Enfermagem - Instituto Federal de Educação, Ciência e Tecnologia de Pernambuco IFPE - Campus Pesqueira, PE, Brasil. E-mail: amgo@discente.ifpe.edu.br

3. ORCID: https://orcid.org/0000-0003-0635-1293. Graduanda em Bacharelado em Enfermagem - Instituto Federal de Educação, Ciência e Tecnologia de Pernambuco IFPE - Campus Pesqueira, PE, Brasil. E-mail: clch@discente.ifpe.edu.br

4. ORCID: https://orcid.org/0000-0002-4520-3536. Graduanda em Bacharelado em Enfermagem - Instituto Federal de Educação, Ciência e Tecnologia de Pernambuco IFPE - Campus Pesqueira, PE, Brasil. E-mail: gss20@discente.ifpe.edu.br

5. ORCID: https://orcid.org/0000-0002-8067-2880. Graduando em Bacharelado em Enfermagem - Instituto Federal de Educação, Ciência e Tecnologia de Pernambuco IFPE - Campus Pesqueira, PE, Brasil. E-mail: jipb@discente.ifpe.edu.br

6. ORCID: https://orcid.org/0000-0002-4947-3787. Graduanda em Bacharelado em Enfermagem - Instituto Federal de Educação, Ciência e Tecnologia de Pernambuco IFPE - Campus Pesqueira, PE, Brasil. E-mail: mftlo@discente.ifpe.edu.br

7. ORCID: https://orcid.org/0000-0003-4119-7076. Mestre. Docente do Instituto Federal de Educação, Ciência e Tecnologia de Pernambuco IFPE - Campus Pesqueira. PE, Brasil. E-mail: ana.sa@pesqueira.ifpe.edu.br

CONTATO: Autor correspondente: Celia Maria Ribeiro de Vasconcelos | Endereço: Estrada do Encanamento n ${ }^{\circ} 122$ Apto 102 Parnamirim, Recife, Pernambuco, Brasil. CEP: 52060-210 | Telefone: (81) 999723702 | E-mail: celia@pesqueira.ifpe.edu.br 
RESUMO Os alunos do Curso de Bacharelado em Enfermagem no Estado de Pernambuco descrevem através de relatos de experiência as dificuldades e mudanças enfrentadas e seus sentimentos em relação ao método de ensino remoto adotado pela instituição durante a pandemia da COVID-19. O relato visa expor as adversidades, problemas e sentimentos que os alunos passaram a enfrentar após o Instituto precisar aderir ao formato de aulas remotas para dar continuidade ao ensino durante $o$ atual contexto que estamos vivenciando. As dificuldades foram apresentadas mediante os relatos individuais de cinco alunos entre agosto a outubro de 2020 , que descreveram os obstáculos e sofrimentos nesse período de isolamento social durante a pandemia. Visando a preservação das suas identidades, esses alunos foram identificando através das cores: Azul; Amarelo; Vermelho; Verde e Branco. Por meio dos relatos individuais de cada aluno foi possível perceber que os principais sentimentos e contrariedades vivenciados pelos mesmos nesse período pandêmico e de aulas online foram: ansiedade, dificuldades para administrar o tempo provocando estresse e problemas com o sono. Por fim, observou-se que a ansiedade e suas consequências foram apontadas pelos estudantes como as principais dificuldades enfrentadas nesse período pandêmico.

DESCRITORES: Ansiedade. Alunos. Covid-19. Ensino Remoto. Pandemia.

ABSTRACT Students of the Bachelor's Degree in Nursing in the State of Pernambuco describe, through experience reports, the difficulties and changes they faced and their feelings in relation to the remote teaching method adopted by the institution during the COVID-19 pandemic. The report aims to expose the adversities, problems and feelings that students started to face after the Institute needed to adhere to the remote classes format in order to continue teaching during the current context we are experiencing. The difficulties were presented through the individual reports of five students between August and October 2020, who described the obstacles and sufferings in this period of social isolation during the pandemic. Aiming at preserving their identities, these students were identified using the colors: Blue; Yellow; Red; Green and White. Through the individual reports of each student it was possible to notice that the main feelings and setbacks experienced by them in this pandemic period and online classes were: anxiety, difficulties in managing time causing stress and problems with sleep. Finally, it was observed that anxiety and its consequences were identified by students as the main difficulties faced in this pandemic period.

DESCRIPTORS: Anxiety. Students. Covid-19. Remote Teaching. Pandemic. 


\section{INTRODUÇÃO}

m dezembro de 2019 em Wuhan, na China, ocorreu a primeira transmissão por um

novo vírus, disseminada e transmitida de pessoa a pessoa, uma doença que mais tarde seria mundialmente conhecida como COVID-19, denominado cientificamente por SARS-CoV-21.

A doença apresenta quadros variáveis de infecções assintomáticas a quadros mais graves, podendo apresentar sintomas que variam entre um leve resfriado a uma Síndrome Gripal-SG "que se caracteriza por, pelo menos dois destes sintomas: sensação febril sendo associada ou não a dor de garganta, dor de cabeça, tosse e coriza" até uma pneumonia severa ${ }^{1}$.

No Brasil a pandemia de COVID-19 trouxe grandes transtornos em todos os aspectos da vida da população brasileira, como: economia, transportes, saúde e na educação. Na tentativa de amenizar o contágio foram adotadas medidas de prevenção sugeridas pela Organização Mundial da Saúde - OMS tais como, lavar as mãos com água e sabão, ou higienizar com álcool em gel a 70\%, ao tossir ou espirrar, cobrir nariz e boca com lenço ou com a parte interna do cotovelo, usar a máscaras em todos os ambientes ao sair de casa, manter distância de 1 (um) metro entre pessoas em lugares públicos e respeitar o isolamento social².

Diante desse contexto, surgiu a possibilidade da realização das aulas utilizando a forma de ensino remoto, que tem como base aulas síncronas, sendo essas de forma online ao vivo e as assíncronas, que constituem na realização de atividades como: leitura prévia dos assuntos e compartilhamento dos trabalhos em alguma plataforma digital para serem executadas individualmente, onde os alunos e os professores pudessem realizar essas atividades de ensino em suas casas. Com a adoção dessa modalidade de ensino, ocasionou o aparecimento de transtornos psicológicos entre os estudantes, em consequência deles se verem obrigados a utilizarem novas metodologias de ensino, completamente diferentes daquelas que as instituições costumavam utilizar ${ }^{3}$.

Os efeitos da pandemia vão além das mortes e do número de infectados, com a implementação do isolamento social foi registrado as seguintes consequências: aumento de casos de ansiedade e estresse na população em geral, comprovado aumento de $32 \%$ nos casos de suicídio, ansiedade que aumentaram de $8,7 \%$ para $14,9 \%$ e os de estresse aumentaram de $6,9 \%$ para $9,7 \%{ }^{4}$. De acordo com uma pesquisa realizada por uma organização estadudense que aponta para os universitários, o desequilíbrio psicológico e as consequências negativas que o distanciamento pode ocasionar na sua vida acadêmica sobressai em relação ao contexto normal ${ }^{5,6}$.

Os problemas com o estresse e as crises de ansiedade ocasionam grandes desafios para a ciência, considerando que, agora a preocupação não está voltada exclusivamente 
para a cura do Coronavírus, mas também direcionada a procurar os meios para evitar ou diminuir os transtornos que o isolamento social tem provocado, considerando que essas perturbações podem contribuir para o aparecimento de resultados mais graves que o da própria COVID-197,8.

A pandemia poderá causar uma condição emocional conturbada referenciado por diversos países onde foi evidenciado, um elevado aumento dos problemas de saúde mental, com destaque para a ansiedade, depressão e o estresse, além de mal-estar causado pelo medo, falta de sono, ocasionando o uso de medicamentos e o registro para comportamentos suicidas ${ }^{9,10}$. Uma organização britânica que tem por objetivo, a luta pela saúde mental de crianças e jovens, realizou uma pesquisa onde pode evidenciar que $83 \%$ dos jovens entrevistados afirmaram que a pandemia contribuiu para o agravo de problemas já existentes relacionados a saúde mental, em decorrência as desconjunturas da rotina acadêmica e social ${ }^{11}$. A ausência de comunicação humana se associa a sequelas negativas na saúde, podendo ocasionar inclusive a morte ${ }^{12}$.

Este artigo de relato de experiência teve por objetivo, descrever os principais sentimentos vivenciados por um grupo de alunos do II módulo do Curso de Bacharelado em Enfermagem de uma Instituição Federal de Ensino no Agreste do Estado de Pernambuco, que interferiu diretamente durante o processo de aprendizagem, por meio da realização do ensino na modalidade remota durante a pandemia pela COVID-19.

\section{MÉTODO}

Esse relato foi construído através da experiência de cinco alunos do Curso de Bacharelado de Enfermagem de uma Instituição Federal de Ensino no Estado de Pernambuco entre o período de agosto a outubro no ano de 2020. Visando a preservação das suas identidades, os alunos foram identificados através das cores: Azul; Amarelo; Vermelho; Verde e Branco.

Baseado nos encontros síncronos e assíncronos vivenciados durante a disciplina Programa Tutorial II, que aconteceram entre 18 de agosto à 1 de setembro de 2020, foram realizados encontros síncronos de cerca de 1 hora utilizando a plataforma Google Meet onde os alunos compartilharam suas opiniões e explicavam suas adaptações e experiências, também foram disponibilizados pelos professores, alguns artigos através do Google Classroom, constituindo esse, o Ambiente Virtual colaborativo de Aprendizagem utilizado. Que através da leitura desses materiais disponibilizados, possibilitou aos alunos, conhecer sobre o ensino remoto e as metodologias ativas de ensino-aprendizagem.

O objetivo desses encontros foi a elaboração de um artigo de relato de experiência, onde cada aluno produziu um Memorial de Trajetória, onde descreveu as observações e sentimentos mais relevantes durante a pandemia da COVID-19, destacando as reflexões 
e dificuldades de adaptação que interferiram no processo de aprendizagem vivenciados pelos estudantes e referenciados no presente estudo. Aconteceram encontros extras além dos já programados para a disciplina com o intuito de orientar, discutir, avaliar e corrigir o andamento da produção do corrente artigo.

Vale ressaltar, que por se tratar de um artigo de relato de experiência, onde os autores do presente artigo, são os indivíduos envolvidos no processo, relatando as suas vivências. Não houve a necessidade do mesmo ser submetido a uma avaliação pelo Comitê de Ética em Pesquisa, considerando que, as referências e os relatos apresentados são de autoria própria dos autores desse artigo.

\section{RESULTADOS E DISCUSSÃO}

O ensino remoto durante esse momento pandêmico levou os alunos a experimentarem diferentes sensações e sentimentos. Por ser um formato de ensino pouco comum, os estudantes passaram a lidar com vários obstáculos para se adequarem, tais como: medo de acessar as aulas, sensação de utilizar as tecnologias inadequadamente, além da ansiedade quanto ao desempenho no processo de aprendizagem. Os professores precisaram também se adaptarem na utilização de novas metodologias ativas no ensino e a se capacitarem para procurar fazer mais e melhor utilizando os recursos tecnológicos. Vale ressaltar que também houve mudanças nos comportamentos das famílias, dos alunos, e nas instituições, ocasionados pelas incertezas e inseguranças provocados por esse período atípico.

Como foi referenciado, as vivências e as experiências dos alunos na maioria das vezes não têm sido satisfatórias, visto que, essa modalidade de ensino trouxe alguns empecilhos que somados a pandemia tornaram um grande problema. Resultando assim, em sentimentos frustrantes.

A seguir discorreremos os relatos de cada aluno, identificado através das cores "azul; amarelo; vermelho; verde e branco" para melhor entendimento e preservação das suas identidades.

Depois de algumas semanas seguindo um cronograma meio louco, de adaptar minha rotina em casa, minhas obrigações domésticas e as obrigações da faculdade, comecei a me sentir esgotada e sem ânimo para nada, o cansaço e a ansiedade passaram a permear todos os dias (Aluno/a azul).

A situação exposta no relato exemplifica o que a Diretoria de Tecnologias na Educação afirma, que com o ensino remoto também afeta os alunos, nos seus estudos e em seu aspecto psicológico. Por não conseguirem se concentrar ao estudar em casa ou se desassociar das distrações externas que o ambiente domiciliar proporciona, perdem 0 foco nas aulas ${ }^{13}$. 
Passar mais tempo em casa o que só fez elevar o nível de ansiedade e insônia constante com várias noites que não foram bem dormidas, com a retomada do semestre de onde paramos na faculdade, tudo só fez piorar ainda mais. $A$ ansiedade é constante, junto dela ainda tem o sentimento de insuficiência, ociosidade e angústia que são frequentes (Aluno/a amarelo).

A quantidade de atividades e a falta de intimidade com o Ensino a Distância prejudicou bastante o psicológico dos alunos, causando a eles sensação de insuficiência e episódios de crise de ansiedade junto a noites de insônia. A cobrança exercida sobre si mesmos no intuito de evitar falhas prejudicou bastante a concentração e desempenho de alguns. O medo de falhar e a pressão sobre si mesmos é a maior inimiga nesse momento tão difícil (Aluno/a vermelho).

Os sentimentos retratados acima convergem com o que foi referenciado no artigo que aborda "aulas remotas e os desafios de professores e alunos", onde ficou evidente que a paralisação do ensino presencial em todas as escolas, tanto públicas como privadas, atingiu pais, alunos, professores e toda a comunidade escolar e em todos os níveis de ensino. Situação essa que interfere na aprendizagem, desejos, sonhos e perspectivas de muitos discentes, provocando um sentimento de adiamento de todos os planos no contexto educacional. Vale salientar que essa mudança gerou uma interferência na vida familiar de todos os familiares, provocando variações de rotinas de trabalho e ocupações ${ }^{14}$.

A ansiedade já era presente antes da pandemia, durante ela, só fez aumentar (Aluno/a verde).

Diante dos relatos discorridos acima percebe-se que a ansiedade foi o sentimento predominante em todos os alunos, pela incerteza do futuro acadêmico e como ele ocorreria, essas mudanças emocionais não foram observadas apenas pelos alunos mas também por estudiosos do mundo todo que constataram o aumento de depressão, estresse e ansiedade nos universitários durante esse período.

(...) com a grande quantidade na demanda de atividades o aumento de estresse, ansiedade, insônia, sensação de insuficiência e incapacidade por não estar conseguindo conciliar os afazeres domésticos e ter que realizar as atividades assíncronas no prazo estimado, foi inevitável (Aluno/a verde).

A ansiedade afeta o bem-estar físico e mental dos alunos que traz consigo a insônia e a dificuldade de uma boa noite de sono, dessa forma pode provocar diversos outros problemas como: enxaqueca e irritabilidade prejudicando o bom desempenho desses alunos em sua vida acadêmica, trazendo uma sensação de insuficiência e incapacidade ${ }^{9}$. 
Esse modo remoto de ensino exige muito mais do aluno e por estar em casa e ter que assumir responsabilidades para com ela, devido a isso, o tempo e concentração dado para as atividades muitas vezes não é o ideal (...) (Aluno/a verde).

Confesso que não consegui me adaptara essas aulas remotas, a sobrecarga de atividades, a dificuldade para acompanhar as aulas e os conteúdos, o projeto de extensão em que eu participava na faculdade que com o decorrer desses acontecimentos passei a não dar a atenção que era fundamental (...) (Aluno/a branco).

Devido à paralisação das aulas e da adesão do modo de ensino remoto, os alunos tiveram que lidar, além da carga e da demanda acadêmica, com os deveres domésticos e devido às dificuldades financeiras em casa, alguns alunos tiveram que procurar emprego e isso afetou na organização e no planejamento do tempo que precisa ser dedicado as aulas síncronas e as atividades assíncronas. Os alunos precisam ter criatividade para reformular a sua rotina e priorizar as atividades mais urgentes ${ }^{15}$.

O ambiente em casa não favorece para que eu tenha um tempo fixo para fazer as atividades e até mesmo para estudar, fico procrastinando o início dos afazeres da faculdade, mas como tenho um prazo a cumprir acabo fazendo uma hora (Aluno/a amarelo).

O ambiente domiciliar que não era tão favorável para esse novo modelo de ensino, pois mesmo sem querer alguém da família acaba interrompendo para pedir algo ou nos momentos de encontros síncronos em que era preciso ligar a câmera e o microfone, alguma pessoa de casa acabavam fazendo barulho (Aluno/a branco).

Ao estar estudando em casa os alunos precisam, além de administrar bem seu tempo, ter um ambiente ao qual seja favorável a concentração nos estudos e também que seus familiares estejam cientes de modo a não atrapalhar esse momento com barulhos ou interrupções desnecessárias ${ }^{16}$, o que não ocorreu com a maioria dos nossos alunos, eles não possuem um ambiente propício aos estudos e isso atrapalhou bastante ao entendimento e a concentração na hora de ouvir e participar dos encontros síncronos e durante a realização das atividades assíncronas.

Apesar das realidades de cada um dos alunos (diferir), o ensino remoto inserido nesse período pandêmico afetou igualmente a todos, originando problemas parecidos em todos os alunos como o aumento do estresse, ansiedade, insônia, sensação de insuficiência e incapacidade.

É importante ressaltar que além de tratamentos específicos, se faz necessário, que os alunos procurem meio de lazer e entretenimento visando espairecer e esvaziar a mente, 
mesmo sabendo da necessidade de priorizar não somente as obrigações, como também a sua saúde mental.

Por fim, é importante destacar que em tempos como esse de isolamento social, ninguém vai estar $100 \%$ adaptado a essas experiências desgastantes em um cenário tão preocupante onde o mundo se encontra, mas é necessário perceber que podemos arrumar jeitos de tratarmos isso em equipe, onde alunos e professores se deem as mãos para juntos, fazerem dessa nova realidade uma experiência mais plena e saudável.

\section{CONSIDERAÇÕES FINAIS}

Diante do cenário atual vivenciado, as angústias e as novas experiências que estamos predestinados a passar, mesmo que os professores e gestores do curso auxilie e preste o apoio necessário aos alunos, a realidade individual poderá influenciar muito na forma de lidar com esses problemas emocionais.

Também é importante destacar que o início de uma adaptação raramente não é fácil nem indolor, visto que, sair da zona de conforto para desbravar novos lugares é sempre uma caminhada árdua até se poder atingir um patamar de conforto novamente. E é nesse momento em que mudanças constantes acontecem, que deve acontecer o desenvolvimento de mecanismos que possam contribuir para a superação desses empecilhos durante o processo de aprendizagem ou ao menos, possa deixar o caminho menos tortuoso.

Além dessas estratégias, é válido lembrar que, algumas vezes a adoção de estratégias como: planejamento semanal, respeitando os prazos e horários estabelecidos para a realização das atividades, estabelecendo um tempo para a realização de atividades físicas, considerando o benefício dos exercícios físicos para a diminuição do estresse e da ansiedade. Agregando ainda a esse planejamento, deve ser estabelecido, tempo para pausas, realizar refeições e estabelecer horário para dormir, o que irá contribuir para garantir a obtenção de uma boa nutrição e proporcionar o descanso almejando e uma melhora no desempenho. Esses benefícios poderão contribuir para reduzir ou eliminar tais sentimentos como, a sensação de insuficiência, incapacidade e tristeza.

\section{REFERÊNCIAS}

1. Brasil. Sobre a Doença Coronavírus [Internet]. Ministério da Saúde; 2020 [acesso em 2020 Set 01]. Disponível em: https://coronavirus.saude.gov.br/sobre-a-doenca

2. Câmara PHS. Decreto $n^{\circ} 48.809$, de 14 de março de 2020 [Internet]. Diário Oficial do Estado de Pernambuco [acesso em 2020 Out 06]. Disponível em: https://www.legisweb.com.br/legislacao/?id=390754

3. Vieira KM, Postiglioni GF, Donaduzz G, Porto CS, Klein LL, Vida de Estudante Durante a Pandemia: Isolamento Social, Ensino Remoto e Satisfação com a Vida [Internet]. Revista Cientifica em Educação a Distância. 2020 [acesso em 2020 Set 29]. Disponível em: https://eademfoco.cecierj.edu.br/index.php/ Revista/article/view/1147/574 
4. Paranashop. Aumento de $32 \%$ de suicídios durante a quarentena alerta para preocupação com a saúde mental na sociedade [Internet]. Paranashop; 2020 [acesso em 2020 Set 01]. Disponível em: https:// paranashop.com.br/2020/07/aumento-de-32-de-suicidios-durante-a-quarentena-alerta-para-preocupacao-com-a-saude-mental-na-sociedade

5. American College Health Association. American College Health Association: National College health assessment II: Reference group executive summary spring 2019 [Internet]. American College Health Association. 2019 [acesso em 2020 Out 06]. Disponível em: https://www.acha.org/documents/ncha/NCHA-II_SPRING_2019_UNDERGRADUATE_REFERENCE\%20_GROUP_EXECUTIVE_SUMMARY.pdf

6. Blakemore SJ, Mills KL. Is adolescence a sensitive period for sociocultural processing? [Internet]. Annual Review of Psychology, (65) 187-207. 2014 [acesso em 2020 Set 29]. Disponível em: https:// www.semanticscholar.org/paper/ls-adolescence-a-sensitive-period-for-sociocultural-Blakemore-Mills/ c277a75901e82be8280142bf9dc69bf3ca3e7d02

7. Wickens CM. The academic and psychosocial impact of labor unions and strikes on university campuses. In M.E. Poulsen (Ed.), Higher education: Teaching, internationalization and student issues. Nova Scotia Publishers; 2011. p. 107-133). Disponivel em: https://www.researchgate.net/publication/288127874_ The_academic_and_psychosocial_impact_of_labor_unions_and_strikes_on_university_campuses

8. Schmidt B, Crepaldi MA, Bolze SDA, Neiva-Silva L, \& Demenech LM. Impactos na Saúde Mental e Intervenções Psicológicas Diante da Pandemia do Novo Coronavírus (COVID-19) [Internet]. Scielo Preprints. Versão 1. 6(37); 1-14. 2020 [acesso em 2020 Set 29]. Disponível em: http://dx.doi.org/10.1590/SciELOPreprints.58

9. Cespedes MS, Souza JCRP. Hábitos e distúrbios do sono nos estudantes de medicina da Universidade Estadual de Mato Grosso do Sul. Arq Med Hosp Fac Cienc Med Santa Casa São Paulo. 66(2); 2020. p.116-123. doi: https://doi.org/10.26432/1809-3019.2020.65.001

10. Gritsenko V, Skugarevsky O, Konstantinov V, Khamenka N, Marinova T, Reznik A, et al. COVID 19 fear, stress, anxiety, and substance use among Russian and Belarusian university students. International Journal of Mental Health and Addiction [Internet]. 2020 [acesso em 2020 Set 29]. doi: https://doi.org/10.1007/ s11469-020-00330-z

11. Youngminds. Coronavirus: Impact on young people with mental health needs [Internet]. 2020 [acesso em 2020 Out 06]. Disponível em: https://youngminds.org.uk/media/3708/coronavirus-report_march2020.pdf

12. Baumeister RF, Leary MR. The need to belong: desire for interpersonal attachments as a fundamental human motivation [Internet]. Psychological Bulletin; 1995 [acesso em 2020 Set 29]. (117) 497-529. Disponível em: https://psycnet.apa.org/record/1995-29052-001.

13. Redação. O ensino remoto e a saúde psicológica de alunos e professores [Internet]. Diretoria de Tecnologias na Educação; 2020 [acesso em 2021 Jul 13]. Disponível (em cache): http://eadparavc.dted.ufma. $\mathrm{br} / \mathrm{p}=2430$

14. Miranda KKCO, Lima AS, Oliveira VCM, \& Telles CBS. Aulas Remotas em tempo de Pandemia: desafios e percepções de professores e alunos [Internet]. Editora Realize; 2020 [acesso em 2021 Jul 13]. Disponível em: https://editorarealize.com.br/editora/anais/conedu/2020/TRABALHO_EV140_MD1_SA_ ID5382_03092020142029.pdf

15. Andrade JG, TIAGO RA. A busca: alcance sucesso profissional transformando sua vida pessoal. Barra Bonita: Solidum, 2006.

16. Unopar. 5 desafios de estudar em casa e como superar cada um deles [Internet]. Unopar; 2020 [acesso em 2020 Nov 20]. Disponível em: https://blog.unopar.com.br/desafios-de-estudar-em-casa/ 